See discussions, stats, and author profiles for this publication at: https://www.researchgate.net/publication/224355450

\title{
Ultrasound imaging media layer texture analysis of the carotid artery
}

Conference Paper · November 2008

DOI: 10.1109/BIBE.2008.4696801 · Source: IEEE Xplore

CITATIONS

READS

0

39

6 authors, including:

Christos P Loizou

University of Cyprus

155 PUBLICATIONS 1,820 CITATIONS

SEE PROFILE

Marios Pantzaris

Cyprus Institute of Neurology and Genetics

176 PUBLICATIONS 2,121 CITATIONS

SEE PROFILE

Ardrew Nicolaides

St George's, University of London/University of Nicosia Medical Schoool, Nicosia, Cy...

875 PUBLICATIONS 27,237 CITATIONS

SEE PROFILE

Some of the authors of this publication are also working on these related projects:

Project Linked 2 Safety View project

Project Using autologous vein View project 


\title{
Ultrasound Imaging Media Layer Texture Analysis of the Carotid Artery
}

\author{
C. P. Loizou, Member, IEEE, M. Pantziaris, A. Nicolaides, A. Spanias, Fellow, IEEE, M.S. Pattichis, \\ Member, IEEE, and C. S. Pattichis, Senior Member, IEEE
}

\begin{abstract}
The intima-media thickness (IMT) of the common carotid artery (CCA) is widely used as an early indicator of cardiovascular disease (CVD). It was proposed but not thoroughly investigated that the media layer (ML) its composition and texture may be indicative for identifying the risk of stroke and differentiating between patients of high and low risk. In this study we investigate the usefulness of texture analysis of the ML of the CCA. The study was performed on 100 longitudinal ultrasound images acquired from asymptomatic subjects at risk of atherosclerosis. The images were separated into three different age groups, namely below 50, 50 to 60, and above 60 years old. A total of 61 different texture features were extracted from the intima-media complex (IMC), ML and the intima layer (IL). The IMC and ML were segmented manually by a neurovascular expert and automatically by a snakes segmentation system. It was shown that texture features extracted from the IL, ML and IMC are significantly different (mean, gray scale median (GSM), standard deviation, contrast, difference variance, periodicity) and that some of them can be associated with the increase (difference variance, entropy) or decrease (GSM) of patient's age. It was also shown that the GSM of the ML falls linearly with increasing media layer thickness (MLT) and with increasing age. Further research is required for estimating other features that may provide information for patients at risk of stroke.
\end{abstract}

\section{INTRODUCTION}

$\mathrm{C}$ ARDIOVASCULAR disease (CVD) is the third leading cause of death and adult disability in the industrial world after hard attack and cancer. According to [1] 80 million American adults have one or more types of CVD of whom about the half are estimated to be age 65 or older. Atherosclerosis causes enlargement of the arteries and thickening of the artery walls and nowadays the intimamedia thickness (IMT) is used as a validated measure for the

Manuscript received July 52008.

C.P. Loizou, is with the Department of Computer Science, School of Sciences, Intercollege, P.O.Box 51604, CY-3507, Limassol, Cyprus (phone: 357-25-381180; e-mail: loizou.c@ lim.intercollege.ac.cy).

M. Pantziaris is with the Cyprus Institute of Neurology and Genetics, Nicosia, Cyprus; (e-mail: pantzari@cing.ac.cy).

A. Nicolaides is with the Vascular Screening and Diagnostic Center, Nicosia, Cyprus; (e-mail: andisnicolai@gmail.com).

A. Spanias, is with the Department of Electrical Engineering, Arizona State University, Arizona, USA; (e-mail: spanias@asu.edu).

M.S. Pattichis, is with the Department of Electrical and Computer Engineering, University of New Mexico, Albuquergue, USA; (email: pattichis@eece.unm.edu).

C.S. Pattichis, is with the Department of Computer Science, University of Cyprus, Nicosia, Cyprus; (e-mail: pattichi@ucy.ac.cy). assessment of atherosclerosis [2] (see Fig. 1). The intima layer (IL) is a thin layer, the thickness of which increases with age, from a single cell layer at birth to $250 \mu \mathrm{m}$ at the age of 40 for non-diseased individuals [3]

The media layer (ML) is characterized by an echolucent region, predominantly composed of smooth muscle cells, enclosed by the intima and adventitia layers (see Fig. 1, band Z6) [2], [4]. It was proposed but not thoroughly investigated, that not only the IMT but rather the ML, its thickness and its textural characteristics may be used for evaluating the risk of a patient to develop a stroke and account in general the risk of the CVD by differentiating between patients at high and low risk for stroke.

While there are several studies reported earlier in the literature showing that the instability of the carotid atheromatous plaque is associated with the plaque characteristics estimated from B-mode ultrasound images [4], [5], there are no other studies reported in the literature where the ML characteristics have been associated with the risk of stroke or with the increase of patients age. While in [4], [5], the echogenecity in atherosclerotic carotid plaques was evaluated through the gray-scale median (GSM) there are very few attempts made to characterize the IL, the ML and the intima media complex (IMC) with a similar gray scale analysis. It is evident from the visual inspections of the IMC in the common carotid artery (CCA) that a great

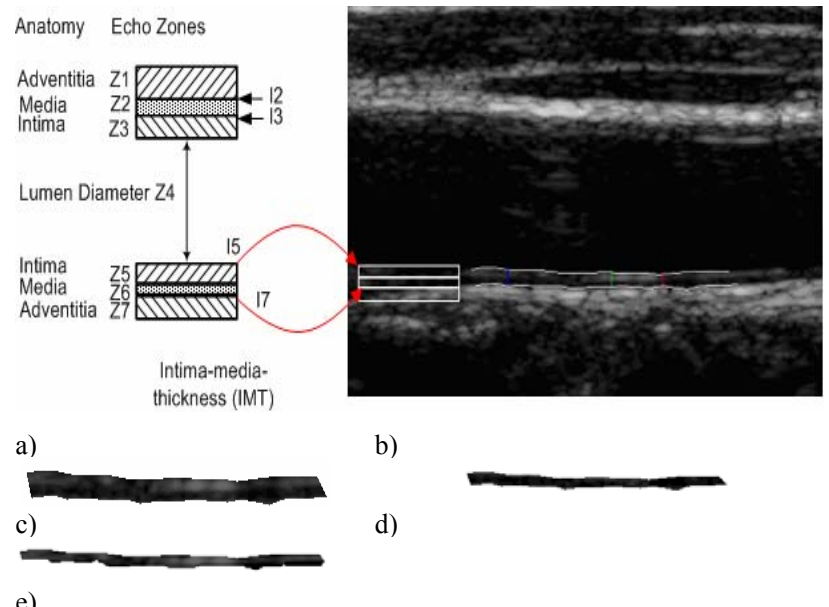

Fig. 1. a) Illustration of the intima-media-complex (IMC) of the far wall of the b) common carotid artery and the automatic IMC segmentation [8], [9]. The media layer (ML) is defined as the layer (band) between the intima-media and the media-adventitia interface (band Z6), c) extracted IMC, d) extracted media layer (ML) and e) extracted intima layer (IL). 
variation in echogenecity does exist. However, the usefulness of this information has not yet been studied.

In [6] the early structural changes of the CCA in familial hypercholesterolemia were investigated by extracting textural characteristics from the IMC, from patients with and without hypercholesterolemia. It was shown that their textural characteristics were significantly different.

Earlier research [7], showed that the media layer thickness (MLT) does not change significantly with age $(125 \mu \mathrm{m}<\mathrm{MLT}<350 \mu \mathrm{m})$. It is furthermore proposed but not thoroughly investigated, that not only the IMT but rather the ML (its composition and thickness) may be used for evaluating the risk of a patient to develop a stroke and account in general the risk of the CVD by differentiating between patients with high and low risk and separate them in different age groups. Furthermore, the alteration of these textural characteristics with increase in age may give additional information for characterizing patients in different risk groups. Texture features derived from statistical, model based, and Fourier based methods, have also been used to characterize and classify carotid atheromatous plaques from B-mode ultrasound images [5].

The objective of our study was to investigate whether textural characteristics extracted from the IL, the ML and the IMC of the CCA, segmented by a snakes segmentation system [8], are associated with the increase of age in these patients and if they can identify patients at low and high risk for stroke.

The paper is organized as follows. In section II materials and methods are given, in section III the results are presented and sections IV and V give the discussion and the concluding remarks respectively.

\section{Materials AND Methods}

\section{A. Recording of Ultrasound Images}

A total of $100 \mathrm{~B}$-mode longitudinal ultrasound images of the CCA used for the IL, ML and IMC texture analysis, were recorded by the ATL HDI-3000 ultrasound scanner (Advanced Technology Laboratories, Seattle, USA) with a linear probe (L74), with a recording frequency of $7 \mathrm{MHz}$, a velocity of $1550 \mathrm{~m} / \mathrm{s}$ and 1 cycle per pulse, which resulted to a wavelength (spatial pulse length) of $0.22 \mathrm{~mm}$ and an axial system resolution of $0.11 \mathrm{~mm}$ [9]. Digital images were resized using the bicubic method to a standard pixel density of 16.66 pixels $/ \mathrm{mm}$ (with a resolution of $0.06 \mathrm{~mm}$ ). The images were recorded at the Cyprus Institute of Neurology and genetics, in Nicosia, Cyprus, from 42 female and 58 male asymptomatic patients aged between 26 and 95 years old, with a mean age of 54 years. These subjects were at risk of atherosclerosis but they have not developed clinical symptoms, such as a stroke or a transient ischemic attack. Furthermore, the images were normalized as described in [10]. The grayscale-normalized image was obtained through algebraic (linear) scaling of the image by linearly adjusting the image so that the median gray level value of the blood
TABLE I

TeXture Features (Median(IQR)) For The IL, ML, AND IMC For The AUTOMATED SEGMENTATIONS.

\begin{tabular}{lccc}
\hline \hline & IL & ML & IMC \\
\hline Mean & $35(19.3)$ & $21(25)$ & $33(22)$ \\
Median & $35(18.5)$ & $28(18)$ & $30(21.3)$ \\
STD & $16(6.6)$ & $14(7)$ & $16(5.7)$ \\
Contrast & $52(75)$ & $28(32)$ & $61(57)$ \\
DV & $16(27.8)$ & $81(56)$ & $32(32)$ \\
Complexity & $1704(3175)$ & $6041(6762)$ & $4166(5888)$ \\
ASM & $0.09(0.04)$ & $0.002(0.003)$ & $0.003(0.002)$ \\
Coarseness & $20(14.5)$ & $13(11)$ & $24(11.3)$ \\
SS-TEL & $38(33)$ & $78(53)$ & $56(38)$ \\
Entropy & $5.7(1.15)$ & $6(1.2)$ & $6.6(0.7)$ \\
Roughness & $2.46(0.187)$ & $2.2(0.100)$ & $2.238(0.079)$ \\
Periodicity & $0.8(0.07)$ & $0.9(0.06)$ & $0.8(0.2)$ \\
\hline \hline
\end{tabular}

IQR: Inter-Quartile-Range, DV: Difference variance, ASM: Angular second moment, SS-TEL: SS-texture energy laws

TABLE II

Wilcoxon Rank Sum Test Performed On Texture Features EXTRACTED From THE IL, ML, AND IMC For THE MANUAL (M) AND THE Automated (A) Segmentation Measurements.

\begin{tabular}{|c|c|c|c|c|c|c|}
\hline & \multicolumn{3}{|c|}{ Manual (M) } & \multicolumn{3}{|c|}{ Automated (A) } \\
\hline & $\begin{array}{l}\sum_{1}^{H} \\
\qquad\end{array}$ & 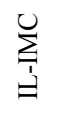 & 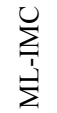 & $\begin{array}{l}\sum_{1}^{H} \\
\Leftrightarrow\end{array}$ & $\begin{array}{l}\sum_{1}^{U} \\
=\end{array}$ & 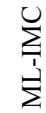 \\
\hline Mean & $\mathrm{S}$ & NS & $\mathrm{S}$ & $\mathrm{S}$ & NS & $\mathrm{S}$ \\
\hline Median & NS & NS & $\mathrm{S}$ & NS & NS & $\mathrm{S}$ \\
\hline Stand. Dev & $\mathrm{S}$ & $\mathrm{S}$ & $\mathrm{S}$ & $\mathrm{S}$ & NS & $\mathrm{S}$ \\
\hline Contrast & $\mathrm{S}$ & $\mathrm{S}$ & $\mathrm{S}$ & $\mathrm{S}$ & NS & $\mathrm{S}$ \\
\hline Difference Var. & $\mathrm{S}$ & $\mathrm{S}$ & S & $\mathrm{S}$ & S & NS \\
\hline Complexity & $\mathrm{S}$ & $\mathrm{S}$ & S & $\mathrm{S}$ & $\mathrm{S}$ & NS \\
\hline ASM & NS & S & NS & $\mathrm{S}$ & $\mathrm{S}$ & $\mathrm{S}$ \\
\hline Coarseness & $\mathrm{S}$ & $\mathrm{S}$ & $\mathrm{S}$ & $\mathrm{S}$ & $\mathrm{S}$ & $\mathrm{S}$ \\
\hline SS-Text.E.L1 & $\mathrm{S}$ & $\mathrm{S}$ & NS & $\mathrm{S}$ & $\mathrm{S}$ & NS \\
\hline Entropy & $\mathrm{S}$ & $\mathrm{S}$ & NS & $\mathrm{S}$ & $\mathrm{S}$ & $\mathrm{S}$ \\
\hline Periodicity & $\mathrm{S}$ & NS & $\mathrm{S}$ & $\mathrm{S}$ & NS & $\mathrm{S}$ \\
\hline
\end{tabular}

TABLE III

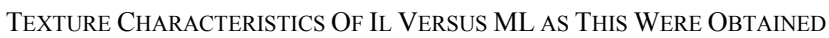
By InTERPRetation Of The TeXture Feature Values Given in TABLE

\begin{tabular}{l|l}
\multicolumn{1}{c}{ IL } & \multicolumn{1}{c}{ ML } \\
\hline Brighter & More dark \\
Higher contrast & Less contrast \\
Slightly more rough & More Smooth \\
Less periodical, more & More periodical, more \\
heterogeneous & homogeneous \\
More coarse, i.e. large areas with & Less coarse, i.e. less local \\
small gray tone variations & uniformity in intensity \\
\hline \hline
\end{tabular}

was $0-5$, and the median gray level of the adventitia (artery wall) was 180-190.

The images were separated into three different age groups depending on age, namely below 50,50 to 60 , and above 60 years old, with 27,36 and 37 patients in each group respectively.

\section{B. Manual Measurements}

A neurovascular expert delineated manually (using the mouse) the IL, ML layer [8] and the IMC [9] on 100 longitudinal ultrasound images of the CCA after image normalization by selecting $20-40$ consecutive points for the 
TABLE IV

MANN-Whitney RANK Sum TeSt PERFormed on TEXTURe Features EXTRACTED FROM THE AUTOMATICALLY SEGMENTED IL/ML/IMC AT $\mathrm{P}<0.05$ For DiFFERENT AGE GROUPS.

\begin{tabular}{|c|c|c|c|c|}
\hline $\begin{array}{l}\text { Texture } \\
\text { Features } \\
\end{array}$ & Age & $50<=$ & 50 to60 & $>60$ \\
\hline \multirow{3}{*}{ GSM } & $50<=$ & $\mathrm{x}$ & $\mathrm{NS} / \mathrm{S} / \mathrm{NS}$ & $\mathrm{NS} / \mathrm{S} / \mathrm{S}$ \\
\hline & 50 to60 & $\mathrm{x}$ & $\mathrm{x}$ & S/NS/NS \\
\hline & $>60$ & $\mathrm{x}$ & $\mathrm{x}$ & $\mathrm{x}$ \\
\hline \multirow{3}{*}{ STD } & $50<=$ & $x$ & NS/NS/NS & $\mathrm{S} / \mathrm{S} / \mathrm{S}$ \\
\hline & 50 to 60 & $\mathrm{x}$ & $\mathrm{x}$ & $\mathrm{NS} / \mathrm{S} / \mathrm{S}$ \\
\hline & $>60$ & $\mathrm{x}$ & $\mathrm{x}$ & $\mathrm{x}$ \\
\hline \multirow{3}{*}{ Contrast } & $50<=$ & $\mathrm{x}$ & NS/NS/NS & S/NS/NS \\
\hline & 50 to 60 & $\mathrm{x}$ & $\mathrm{x}$ & NS/NS/NS \\
\hline & $>60$ & $\mathrm{x}$ & $\mathrm{x}$ & $\mathrm{x}$ \\
\hline \multirow{3}{*}{ DV } & $50<=$ & $\mathrm{x}$ & NS/NS/NS & $\mathrm{NS} / \mathrm{S} / \mathrm{S}$ \\
\hline & 50 to 60 & $\mathrm{x}$ & $\mathrm{x}$ & NS/NS/NS \\
\hline & $>60$ & $\mathrm{x}$ & $\mathrm{x}$ & $\mathrm{x}$ \\
\hline \multirow{3}{*}{ Complexity } & $50<=$ & $\mathrm{x}$ & NS/NS/NS & $\mathrm{S} / \mathrm{S} / \mathrm{NS}$ \\
\hline & 50 to60 & $\mathrm{x}$ & $\mathrm{x}$ & NS/S/NS \\
\hline & $>60$ & $\mathrm{x}$ & $\mathrm{x}$ & $\mathrm{x}$ \\
\hline \multirow{3}{*}{ Coarseness } & $50<=$ & $x$ & NS/NS/NS & NS/S/NS \\
\hline & 50 to 60 & $\mathrm{x}$ & $\mathrm{x}$ & NS/S/NS \\
\hline & $>60$ & $\mathrm{x}$ & $\mathrm{x}$ & $\mathrm{x}$ \\
\hline \multirow{3}{*}{ SS-TEL } & $50<=$ & $\mathrm{x}$ & $\mathrm{NS} / \mathrm{S} / \mathrm{NS}$ & $\mathrm{NS} / \mathrm{S} / \mathrm{NS}$ \\
\hline & 50 to60 & $\mathrm{x}$ & $\mathrm{x}$ & NS/S/NS \\
\hline & $>60$ & $x$ & $\mathrm{x}$ & $\mathrm{x}$ \\
\hline \multirow{3}{*}{ Entropy } & $50<=$ & $x$ & NS/NS/NS & $\mathrm{NS} / \mathrm{NS} / \mathrm{S}$ \\
\hline & 50 to60 & $\mathrm{x}$ & $\mathrm{x}$ & NS/NS/NS \\
\hline & $>60$ & $\mathrm{x}$ & $\mathrm{x}$ & $\mathrm{x}$ \\
\hline
\end{tabular}

STD: Standard Deviation, DV: Difference variance, SS-TEL: SS-texture energy laws, S: Significant difference at $\mathrm{p}<0.05$, NS: Non significant difference at $\mathrm{p}>0.05$

adventitia, media and intima at the far wall of the CCA. The manual delineations were performed using a system implemented in MATLAB $^{\circledR}$ from our group. The measurements were performed between $1-2 \mathrm{~cm}$ proximal to the bifurcation of the CCA on the far wall [2] over a distance of $1.5 \mathrm{~cm}$ starting at a point $0.5 \mathrm{~cm}$ and finishing at a point $2.0 \mathrm{~cm}$ proximal to the carotid bifurcation. The bifurcation of the CCA was used as a guide and all measurements made from that region. The IMT, MLT and the ILT were then calculated as the average of all measurements. The measuring points and delineations were saved for further comparison. All sets of manual segmentation measurements were performed by the expert in a blinded manner, both with respect to identifying the subject and as to the image delineation.

\section{IL, ML and IMC Snake Segmentations}

A total of 100 ultrasound images of the CCA were segmented to identify the IL, ML and IMC. Segmentation was carried out after image normalization using the automated snakes segmentation system proposed and evaluated on ultrasound images of the CCA in [9] and [8], which is based on the Williams \& Shah [11] snake.

Initially the IMC was segmented [9] where the boundaries I5 (lumen-intima interface) and I7 (media-adventitia interface) were extracted. The upper side of the ML (see Fig. 1, Z6) was then estimated by deforming the lumen-intima interface (boundary I5) by $0.36 \mathrm{~mm}$ (6 pixels) downwards and then deformed by the snakes segmentation algorithm proposed in [9] in order to fit to the media boundary. This displacement of $0.36 \mathrm{~mm}$ is based on the observation that the manual mean IMT lies between $0.54 \mathrm{~mm}$ (minimum of $\mathrm{IMT}_{\text {mean }}$ ) and $0.88 \mathrm{~mm}$ (maximum of $\mathrm{IMT}_{\text {mean }}$ ), with a mean IMT of $0.71 \mathrm{~mm}$ [9]. By taking into consideration that the spatial resolution (distance between two pixels) is $0.06 \mathrm{~mm}$, then the IMT is lying within the range of 0.54 $\mathrm{mm}<\mathrm{IMT}<0.88 \mathrm{~mm}(9<\mathrm{IMT}<15$ pixels $)$, with a mean of $0.71 \mathrm{~mm}$ (12 pixels). Therefore the displacement of the contour, in order to estimate the media should be in average $0.36 \mathrm{~mm}$ (6 pixels times $0.06 \mathrm{~mm}$ ) downwards, which is the half of the size of the IMT (the distance between I5 and I7, where $\mathrm{I} 7$ is the media-adventitia interface).

In order to achieve standardization in extracting the thickness and texture measures from IMC, IL and ML segments with similar dimensions, the following procedure was carried out. A region of interest of $9.6 \mathrm{~cm}$ (160 pixels) in length was extracted.

\section{Texture Analysis}

Texture provides useful information for the characterization of the atherosclerotic carotid plaque [4], [5]. In order to estimate textural characteristics extracted from the IL, ML, and the IMC a total of 61 different texture features were extracted both from the manual and the automated segmented regions of interest. The following texture feature set algorithms were used: (i) Statistical Features [8], (ii) Spatial Gray Level Dependence Matrices as proposed by Haralick et al. [12], (iii) Gray Level Difference Statistics [13], (iv) Neighborhood Gray Tone Difference Matrix [14], (v) Statistical Feature Matrix [15], (vi) Laws Texture Energy Measures [15], (vii) Fractal Dimension Texture Analysis [15], and (viii) Fourier Power Spectrum [15].

The Mann-Whitney rank sum test, which calculates the difference between the sum of the ranks of two different independent samples was used in order to identify if for each set of the extracted texture features, a significant difference (SD) or not (NS) exists, $\mathrm{p}<0.05$, for the three different age groups.

The Wilcoxon rank sum test, which calculates the difference between the sum of the ranks of two dependent samples, was also used in order to identify if for each set of the textural characteristics extracted from the IL, ML, and the IMC for both the manual and the automated segmentations, a significant difference (SD) or not (NS) exists, at $\mathrm{p}<0.05$. Furthermore, box plots for the three different age groups, were plotted for different texture features. Finally, regression analysis was also used to demonstrate the relationship between the gray-scale median (GSM) and age and between the GSM and MLT.

\section{RESULTS}

Figure 1 illustrates an original normalized ultrasound image of the CCA with the automated segmentation of the IMC in b) and the extracted IMC, ML and IL in b), c) and d) respectively. 


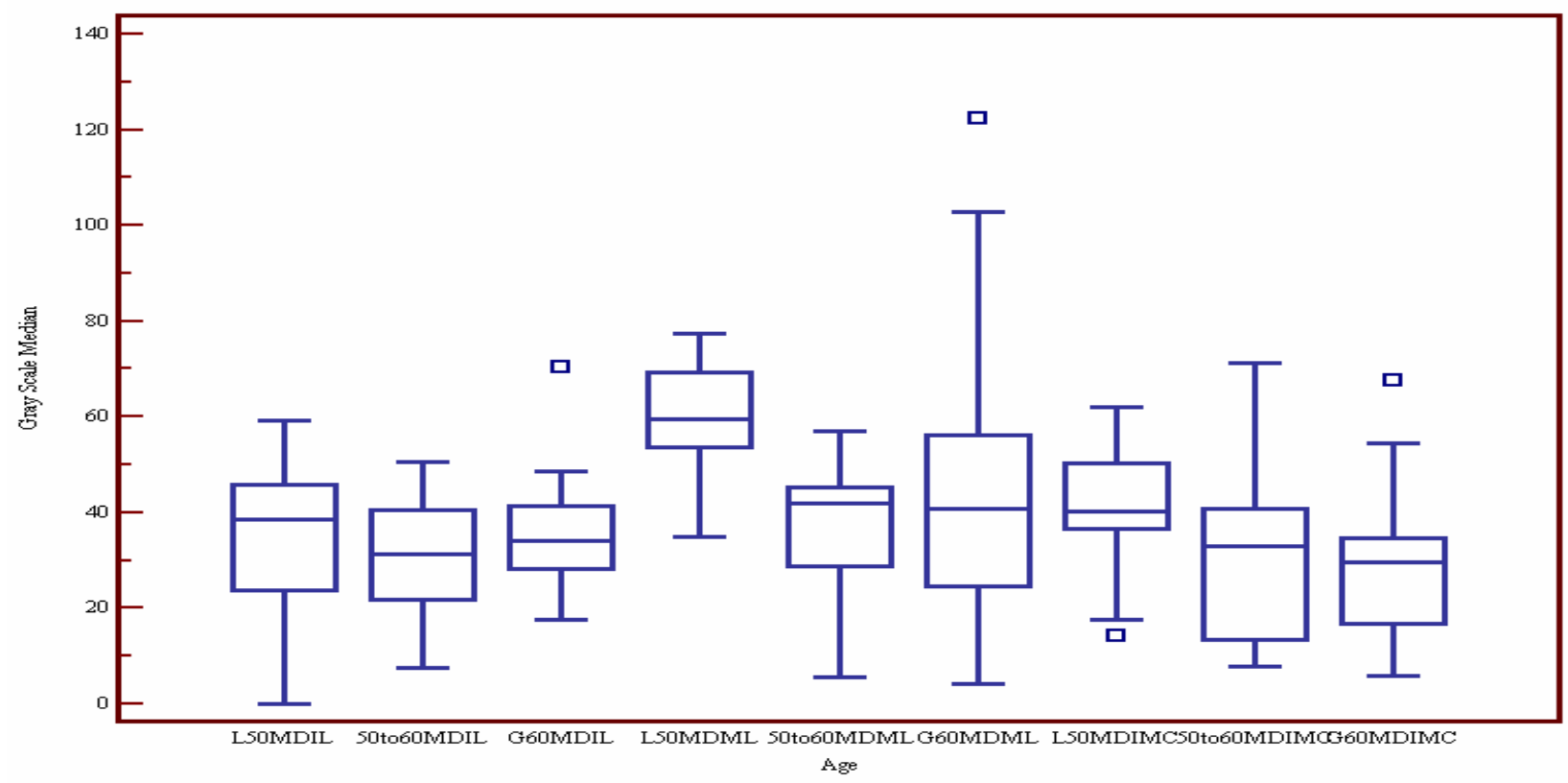

a) IQR: 22, 18.4, 15.4/16, 19.5, 23.7/13.6, 25,2, 16.9

Fig. 2. Box plots of the GSM texture feature, for the age groups below 50 (L50), 50 to 60 (G60), and above 60 years old, extracted from the automated IL, ML and IMC segmentations. The IQR values for the IL/ML/IMC are also shown.

Table I presents selected texture features extracted from the automated (A) IL, ML, and IMC segmentations with their inter-quartile range values (IQR) shown in parentheses. Table I also shows that most of the texture features extracted from the ML exhibit lower median and IQR values when compared with those extracted from the IMC and IL (mean, GSM, STD, contrast, ASM, coarseness).

The Wilcoxon rank sum test performed on selected texture features for the IL, ML, and IMC extracted from all 100 ultrasound images of the CCA for the $\mathrm{M}$ and the A segmentations, is shown in Table II. It is shown that 10 out of 11,5 out 11 , and 3 out of 11 features exhibit SD changes for the automated segmentations between the IL and ML, IL and IMC, and ML and IMC respectively. Similar performance was also obtained for the manual segmentations.

Table III summarizes the texture characteristics of IL versus ML as these were obtained by interpretation of the values of the texture features given in Table I.

Table IV presents the Mann-Whitney rank sum test performed on selected texture features extracted from the automated IL, ML and IMC segmentations for the three different age groups (below 50, between 50 and 60, and over 60 years old). It is shown that some of the texture features (GSM, STD, DV, complexity, coarseness and SS-TEL) are SD between different age groups.

Figure 2 presents box plots of the GSM texture feature, for the three different age groups, extracted from the automated IL/ML/IMC segmentations for all 100 images of the CCA with their inter quartile ranges (IQR).

Figure $3 \mathrm{a}$ presents the results of the patients age versus GSM of the ML using regression analysis with an intercept 57.4 (standard error of 2.29) and a slope -0.26 (standard error of 0.98). The mean square error due to regression was 366, the residual mean square was 399 resulting into an FRatio of 92 with a corresponding significance level of $\mathrm{p}=0.34$. This supports the assumption of a linear relationship between GSM of the ML and patients age. The correlation coefficient was $\rho=0.11$. It is shown that the ML GSM at age 50 years old is 62.2 . Figure $3 \mathrm{~b}$ presents the results of the automated MLT values versus the GSM of the ML using regression analysis with an intercept 46.63 (standard error of 5.45) and a slope -16 (standard error of 19.8). The mean square error due to regression was 262 , the residual mean square was 401 resulting into an F-Ratio of 0.65 with a corresponding significance level of $p=0.42$. This supports the assumption of a linear relationship between ML GSM and MLT. The correlation coefficient was $\rho=0.09$. It is shown that the GSM at MT $=0.3 \mathrm{~mm}$ is 45 . Regression analysis performed, showed that there is a linear relationship between age and these features.

Our study showed (see also Fig. 2 and Fig. 3) that there are some texture features (DV, entropy), which linearly increases with increasing age, while others (GSM) linearly decreases for all measurements of the IL, ML and IMC. The decrease of GSM with increasing age and MLT can be attributed to the fact that, at the initial stages of the atherosclerosis disease there is an increased concentration of lipids and hyperplasia of muscle fibers in the ML, which produces hypoechoic (echolucent) structures.

\section{Discussion}

In this study textural characteristics of the IL, ML and IMC of 100 longitudinal ultrasound images of the CCA of asymptomatic subjects were investigated. It was found (see 


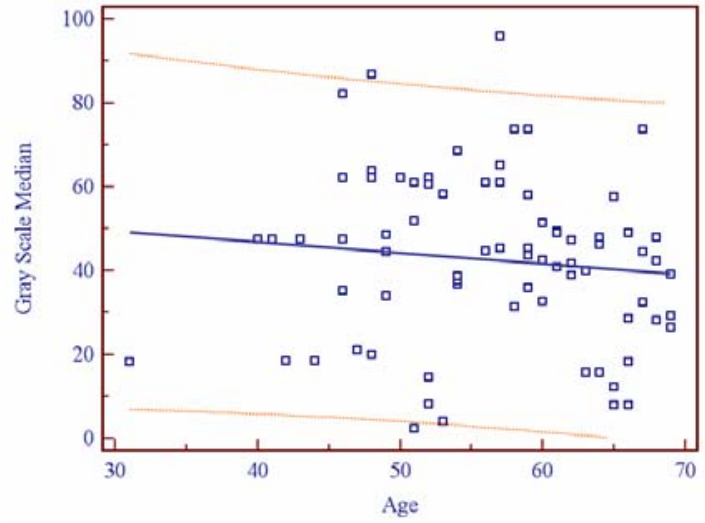

a) $\mathrm{GSM}=57.4-0.26 * \mathrm{Age}$

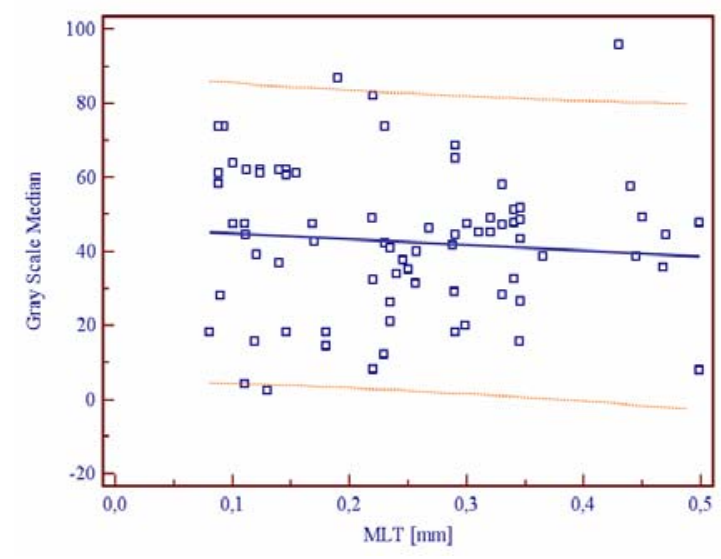

b) $\mathrm{GSM}=46.63-16 * \mathrm{MLT}$

Fig. 3. Decrease of the GSM of the ML with a) Age, with a correlation coefficient $\rho=0.11$, and b) with MLT, with a correlation coefficient $\rho=0.09$.

Table III) that the ML is darker, has less contrast, is more periodic and is less coarse compared to IL.

In [16], where 1016 subjects aged 70 randomly chosen were investigated, it was shown that, the GSM of the IMC of the CCA is closely related to the echogenecity in overt carotid plaques. More specifically it was shown that in subjects with a carotid plaque, IMC GSM in CCA was correlated to GSM in the plaque independently of plaque size and IMT. This finding suggests that the GSM and maybe other texture features extracted from the ML could be important and easily measurable characteristics of the CCA wall that may have prognostic impact for the CVD risk. The IMC-GSM (SD) was 79 (24) and the GSM in plaque was 74 (32), where in our study the IM-GSM was 30 (22).

In [6] the texture features of 12 subjects ( $28 \pm 2$ years) with familial hypercholesterolemia and without CVD, before and 3 months after treatment with atorvastatin were investigated, where the entropy and the ASM were extracted from the IMC band. These were compared with textural characteristics extracted from 12 healthy individuals $(28 \pm 2$ years). It was shown that the extracted texture features for the normal subjects showed significantly higher entropy (normal: $0.82 \pm 0.02$ vs hypercholesterolemia: $0.566 \pm 0.12$ $\mathrm{p}<0.01$ ) and lower ASM (normal: 0.01 \pm 0.01 vs hypercholesterolemia: $0.004 \pm 0.001 \mathrm{p}<0.05$ ) values when compared with the texture features extracted from the patients with hypercholesterolemia. It was finally concluded that in young subjects with early hypercholesterolemia, texture analysis of two-dimensional carotid artery images shows increased IMT and dyshomogeneity, suggestive of early ML infiltration. These alterations are reversed by atorvastatin. Entropy and ASM values reported in our study for the IMC were $6.6(0.7)$ and $0.003(0.002)$ respectively.

In [5], the morphology of atherosclerotic carotid plaque was investigated based on the textural characteristic extracted from 230 ultrasound images of carotid plaque, where it was shown that it is possible to identify a group of patients, symptomatic or asymptomatic, at risk of stroke based on these texture features. It was also shown that there was a significant overlap between the pattern spectra coming from symptomatic and asymptomatic plaques. In our study it was shown (Table IV) also showed that the contrast, which is traditionally used for identifying textural changes [5] is not significantly different between different age groups and thus can not be used for identifying them.

It was furthermore documented in [17], that carotid endarterectomy in asymptomatic individuals with stenosis grater than $70 \%$ reduces the risk of stroke from $2 \%$ per year to $1 \%$ per year. The implication is that approximately 100 operations need to be performed to prevent one stroke. A method based on image analysis of the ML in ultrasound images of the CCA that can calculate and correlate the disease progression from the textural characteristics extracted, has the potential to refine the indications for surgery and spare many patients from an unnecessary and costly operation, which itself carries a $2.7-3.1 \%$ risk of stroke.

In another study [18] where the relationship of the IMT in the CCA and atherosclerosis was investigated on 182 symptomatic patients (mean age 67 years), it was shown that the IMT was correlated to age, male gender, ischemic hard disease, and presence of plaque or stenosis in any of the carotid bifurcations. The results also support earlier observations suggesting faster development of plaques on the left that on the right side of the CCA. It was furthermore shown that the strong correlation between age and IMT was the most important factor for determining the degree of atherosclerosis. Echogenic plaques were more common than echolucent, but the later caused significantly more stenosis. No relationship was found between plaque echogenecity and IMT.

In a recent study [19], where the alterations of the CCA with age in ultrasound images were investigated, it was shown that the diastolic and systolic lumen diameters are increasing with age, thus reducing the wall stress and therefore the elasticity of the wall decreases with age. Furthermore, there was not a well defined boundary found between the age groups below 25, from 26 to 30 and 36 to 40 years old in the pulsatile changes measured. That gave an 
overall idea that the lumen boundary increases with age and the pulsatile variations decrease with increasing age.

While IMT is an established measure of the vascular wall disease [2], GSM is less commonly used while other features have not been used before. GSM analysis has previously mainly been performed on plaques and then found to be related to histological features of the plaque, such as the elastin and calcium content, as well as to the size of the lipid-rich necrotic core [20]. However, the histological correlate to variation in the ML texture features has to be evaluated and the prognosis impact of these new variables has to be investigated.

To the best of our knowledge no other study carried out ML and IL ultrasound textural measurements for investigating their relationship with the increase of age, and the risk of stroke based on their textural characteristics. However, in [4], [5] plaque characteristics were extracted from the carotid plaque, whereas in [17], [21] the variation of the IMT was investigated for different age groups, where it was shown that IMT increases linearly with age.

\section{CONCLUding ReMARKS}

The texture analysis presented in this study, performed on the ML, IL, and the IMC, on 100 ultrasound images of the CCA of asymptomatic subjects, showed that: 1) The ML exhibits higher texture feature values when compared with the IMC, 2) Texture features extracted from the ML and the IMC are mostly SD between ages, 3) Texture features extracted from the $\mathrm{M}$ and the A segmentations are mostly $\mathrm{SD}$, and 4) There is a linear relationship between texture features and age as well as between ML and texture features.

In conclusion the results of this study showed that it is possible to identify a group of patients at risk of atherosclerosis based on their texture features extracted from the ML and the IMC of high-resolution ultrasound images of the CCA. It may also be possible to identify and differentiate those individuals into high and low risk groups according to their cardiovascular risk before the development of plaques. The proposed methodology may also be applied to a group of people, which already developed plaques in order to study the contribution of the ML texture features to cardiovascular risk. Both groups of patients may be benefited by prognosing and managing future cardiovascular events.

Another possible future application of the proposed methodology is that it can to be used in order to investigate the possible effects of statins or other drugs in texture feature changes of the ML of the CCA.

\section{REFERENCES}

[1] American Heart Association, Heart disease and stroke statistics-2007, update. Dallas, Texas, 2007.

[2] P. Pignoli, E. Tremoli, A. Poli, P. Oreste, R. Paoletti, "Intima plus media thickness of the arterial wall: A direct measurement with ultrasound imaging", Atheroscl., vol. 74, no. 6, pp. 1399-1406, 1986.

[3] C.D. Mario, G. Gorge, R. Peters, F. Pinto, D. Hausmann, C. von Birgelen, A. Colombo, H. Murda, J. Roelandt, R. Erbel, "Clinical application and image interpretation in coronary ultrasound. Study group of intra-coronary imaging of the working group of coronary circulation and of the subgroup of intravascular ultrasound of the working group of echocardiography of the European Society of Cardiology", Eur. Heart J., vol. 19, pp. 201-229, 1998.

[4] M.L. Grønhold, B.G. Nordestgaard, T.V. Schroeder, S. Vorstrup, H. Sillensen, "Ultrasonic echolucent carotid plaques predict future strokes", Circulation, vol. 104, no. 1, pp. 68-73, 2001.

[5] C.I. Christodoulou, C.S. Pattichis, M. Pantziaris, A. Nicolaides, "Texture-Based Classification of Atherosclerotic Carotid Plaques," IEEE Trans. Med. Imag., vol. 22, no. 7, pp. 902-912, 2003.

[6] F. Bartolomucci, M. Paterni, C. Morizzo, M. Kozakova, N. D'Allitto, F. Strippoli, C. Palombo, G. Maiorano, "Early structural changes of carotid artery in familial hypercholesterolemia," Am. J. Hypertension, vol. 14, pp. 125A-126A, 2001.

[7] E.J. Gussenhoven, P.A. Frietman, S.H., R.J. van Suylen, F.C. van Egmond, C.T. Lancee, H. van Urk, J.R. Roelandt, T. Stijnen, N. Bom, "Assessment of medial thinning in atherosclerosis by intravascular ultrasound", Am. J. Cardiol., vol. 68, pp. 1625-1632, 1991.

[8] C.P. Loizou, C.S. Pattichis, M. Pantziaris, A. Nicolaides, N. Georgiou, E. Kyriakou, "Media thickness measurement of the common carotid artery," Proc. IEEE EMBS 2007, The29th Annual Int. Conf. IEEE Engin. Med. Biol., Cite Int., Lyon, France, FrP1B6.5, pp. 2171-2174, Aug. 23-26, 2007

[9] C.P. Loizou, C.S. Pattichis, M. Pantziaris, T. Tyllis, A. Nicolaides, "Snakes based segmentation of the common carotid artery intima media," Med. Bio. Eng. Comput.," vol. 45, pp. 35-49, 2007.

[10] T.J. Tegos, M.M. Sabetai, A.N. Nicolaides, T.S. Elatrozy, S. Dhanjil, J.M. Stevens, "Patterns of brain computed tomography infraction and carotid plaque echogenicity", J. Vasc. Sur., vol. 33, pp. 334-339, 2001.

[11] D.J. Williams, M. Shah, "A fast algorithm for active contour and curvature estimation", GVCIP: Image Underst., vol. 55, no. 1, pp. 426, 1992.

[12] R.M. Haralick, K. Shanmugam, and I. Dinstein, "Texture Features for Image Classification," IEEE Trans. Systems, Man., and Cybernetics, vol. SMC-3, pp. 610-621, Nov. 1973.

[13] J.S Weszka., C.R. Dyer, and A. Rosenfield, "A comparative study of texture measures for terrain classification," IEEE Trans. Systems, Man. \& Cybernetics, vol. SMC-6, pp. 269-285, April 1976.

[14] M. Amadasun and R. King, "Textural features corresponding to textural properties," IEEE Trans. Systems, Man, \& Cybernetics, vol. 19, no. 5, pp. 1264-1274, Sept. 1989.

[15] C.M. Wu, Y.C. Chen, and K.-S. Hsieh, "Texture features for classification of ultrasonic images," IEEE Trans. Med. Imaging, vol. 11, pp. 141-152, June 1992.

[16] L. Lind, J. Andersson, M. Roenn, T. Gustavsson, "The echogenecity of the intima-media complex in the common carotid artery is closely related to the echogenecity in plaques", Atherosclerosis, vol. 195, pp. 411-414, 2007.

[17] Prevention of disampling and fatal strokes by successful carotid endarterectomy in patients without recent neurological symptoms: randomized control trial, The Lancet, vol. 363(9420), pp. 1491-1502, May 2004.

[18] S. Rosfors, S. Hallerstam, K. Jensen-Urstad, M. Zetterling, C. Carlstroem, "Relationship between intima-media thickness in the common carotid artery and atherosclerosis in the carotid bifurcation", Stroke, vol. 29, pp. 1378-1382, 1998.

[19] J.K. Balasundaram, R.S.D. Wahida Banu, "A non-invasive study of alterations of the carotid artery with age using ultrasound images", Med. Biol. Eng. Comput., vol. 44, pp. 767-772, 2006.

[20] I. Goncalves, M.W. Lindholm, L.M. Pedro, N. Dias, J. Fernandes, G.N. Fredrikson, J. Nilsson, J. Moses, M.P.S. Ares, "Elastin and calcium rather than collagen or lipid content are associated with echogenecity of human carotid plaques", Stroke, vol. 35, no. 2, pp. 795-800, 2004.

[21] S. Graf, J. Gariery, M. Massonneau, R. Armentano, S. Mansour, J. Barra, A. Simon, J. Levenson, "Experimental and clinical validation of arterial diameter waveform and intimal media thickness obtained from B-mode ultrasound image processing", Ultras. Med. Biol., vol. 25, no. 9, pp. 1353-1363, 1999 . 